\title{
A CONTINUOUS BASIS FOR ORLICZ SPACES
}

\author{
ROBERT E. ZINK
}

1. In 1910 Haar created the orthonormal system that bears his name in order to show that there are $O N$ systems with respect to which the Fourier series of each continuous function converges uniformly to the function. The Haar functions are themselves discontinuous, however, and this led Franklin to construct a continuous $O N$ set that plays the same role. Now the Haar functions comprise a Schauder basis for each of the spaces $L^{p}[0,1], p \geqq 1$, as Schauder himself has shown [5]. Indeed, this system serves as a Schauder basis for each of the separable Orlicz spaces associated with the unit interval, see [2], [4]. Thus, it is natural to inquire whether the Franklin system also serves as a basis for these spaces.

In the present article this question is answered affirmatively. The proof is elementary, requiring only the judicious application of the uniform boundedness principle and the Jensen integral inequality. For the spaces $L^{p}, p \geqq 1$, this result has been noted recently by Ciesielski [1]. Nevertheless, even for this special case, the demonstration given below may be of interest by virtue of its utter simplicity.

2. Let $M$ be an $N$-function and let $L^{M}$ be the class of all measurable functions $f:[0,1] \rightarrow R$ for which $\int_{0}^{1} M(|f(t)|) d t<+\infty$. Denote by $O^{M}$ the Orlicz space engendered by $M$ and let $E^{M}$ be the closure in $O^{M}$ of the set of bounded elements of $L^{M}$. It will suffice to show that the Franklin system is a Schauder basis for $E^{M}$, for in the separable case $E^{M}$ and $O^{M}$ coincide [2].

Define the transformations $T_{n}: C[0,1] \rightarrow C[0,1]$ as follows: $T_{n} f$ $=\sum_{k=1}^{n} c_{k} \phi_{k}$, where $\left\{\phi_{k}: k=1,2, \cdots\right\}$ is the Franklin system and, for each $k$,

$$
c_{k}=\int_{0}^{1} f(t) \phi_{k}(t) d t .
$$

According to Franklin's theorem, $\left\|T_{n} f-f\right\|_{\infty} \rightarrow 0$ for each continuous $f$; hence, $\left\{\left\|T_{n}\right\|\right\}_{n=1}^{\infty}$ is a bounded sequence, by virtue of the uniform boundedness principle. Now

Received by the editors August 26, 1967. 


$$
\begin{aligned}
\left\|T_{n}\right\| & =\sup \left\{\left\|T_{n} f\right\|_{\infty}: f \in C[0,1],\|f\|_{\infty}=1\right\} \\
& =\sup _{\|f\|=1} \sup _{x \in[0,1]}\left|\int_{0}^{1} \sum_{k=1}^{n} \phi_{k}(x) \phi_{k}(t) f(t) d t\right| \\
& =\sup _{\|f\|=1} \sup _{x \in[0,1]}\left|\int_{0}^{1} K_{n}(x, t) f(t) d t\right| \\
& =\sup _{x \in[0,1]} \int_{0}^{1}\left|K_{n}(x, t)\right| d t .
\end{aligned}
$$

There follows the existence of a constant $K$ such that

$$
\sup _{x \in[0,1]} \int_{0}^{1}\left|K_{n}(x, t)\right| d t=\sup _{t \in[0,1]} \int_{0}^{1}\left|K_{n}(x, t)\right| d x \leqq K
$$

for every natural number $n$. Certainly one may assume that $K$ is not less than 1.

The transformations $U_{n}: E^{M} \rightarrow E^{M}$ are the natural extensions of the corresponding mappings $T_{n}$ and are formally defined by the same systems of equations. The heart of the argument is the demonstration of the boundedness of $\left\{\left\|U_{n}\right\|\right\}_{n=1}^{\infty}$. When this has been done, the proof will be very nearly complete.

A not too difficult application of the Jensen inequality is sufficient to show that, for $u$ in $L^{M}$,

$$
M\left(\left|\int_{0}^{1} K_{n}(x, t) u(t) d t\right|\right) \leqq \int_{0}^{1} M(K|u(t)|)\left|K_{n}(x, t)\right| d t
$$

for every $x$ in $[0,1], n=1,2, \cdots$. (A proof of this assertion is embedded in Hilfssatz 1 of [3].) Let $u$ be an element of $E^{M}$ satisfying $\|K u\|_{M} \leqq 1$. Then, for each $x$ in $[0,1]$,

$$
\begin{aligned}
M\left(U_{n} u(x)\right) & =M\left(\int_{0}^{1} K_{n}(x, t) u(t) d t\right) \\
& \leqq M\left(\int_{0}^{1}\left|K_{n}(x, t) u(t)\right| d t\right) \\
& \leqq \int_{0}^{1} M(K|u(t)|)\left|K_{n}(x, t)\right| d t
\end{aligned}
$$

by virtue of the Orlicz lemma. Thus, 


$$
\begin{aligned}
\left\|U_{n} u\right\|_{M} & \leqq \int_{0}^{1} M\left(U_{n} u(x)\right) d x+1 \\
& \leqq \int_{0}^{1} \int_{0}^{1} M(K|u(t)|)\left|K_{n}(x, t)\right| d t d x+1 \\
& \leqq K \int_{0}^{1} M(K|u(t)|) d t+1 \\
& \leqq K\|K u\|_{M}+1 \leqq K+1
\end{aligned}
$$

for every natural number $n$. It follows that $\left\{\left\|U_{n}\right\|\right\}_{n=1}^{\infty}$ is bounded by $K(K+1)$. (Note that this portion of the argument can be greatly simplified if it be assumed that $M$ satisfies the $\Delta_{2}$ condition. Unfortunately, one then obtains a correspondingly weaker result.)

Because the continuous functions constitute a dense subset of $E^{M}$ and because convergence in $E^{M}$ is implied by uniform convergence, it follows that $U_{n} u=\sum_{k=1}^{n} c_{k} \phi_{k}$ converges to $u$ for every $u$ in $E^{M}$.

To demonstrate the unicity of the coefficient sequence, it suffices to note that convergence in the Orlicz norm implies convergence in $L^{1}$, so that from the relation $u=\sum_{k=1}^{\infty} d_{k} \phi_{k}$, one obtains for each $m$,

$$
\begin{aligned}
d_{m} & =\lim _{n} \int_{0}^{1}\left(\sum_{k=1}^{n} d_{k} \phi_{k}(t)\right) \phi_{m}(t) d t \\
& =\int_{0}^{1} u(t) \phi_{m}(t) d t=c_{m} .
\end{aligned}
$$

\section{REFERENCES}

1. Z. Ciesielski, Properties of the orthonormal Franklin system. II, Studia Math. 27 (1966), 289-323.

2. M. A. Krasnosel'skiY and Ya. B. Rutickil, Convex functions and Orlicz spaces, Noordhoff, Groningen, 1961.

3. W. Orlicz, Ein Satz über die Erweiterung von linearen Operationen, Studia Math. 5 (1934), 127-140.

4. - Über eine gewisse Klasse von Raumen vom typus B, Bull. Internat. Acad. Polon. Ser. A Cracovie 8 (1932), 207-220.

5. J. Schauder, Einige Eigenschaft des Haarschen Orthogonalsystems, Math. Z. 28 (1928), 317-320.

PuRdue University 